\title{
Bio-inspired True Coalition Formation on Intrusion Detection by Mobile Robots
}

\author{
Xiannuan Liang and Yang Xiao, Senior Member, IEEE
}

\begin{abstract}
In this paper, we study a true coalition formation of three robots (i.e., 2 robots vs. 1 robot) in order to detect and capture intrusions in a curved loop. A coalition benefits robots by increasing detection and capture strength, but it has a tradeoff of paying an investment cost for each individual. We derive that a true coalition only arises when an interior evolutionarily stable strategy exists. We also find out the conditions under which an interior evolutionarily stable strategy exists.
\end{abstract}

Index items- Robots, Mobile Sensors, Collaboration, Coalition, Game Theory

\section{INTRODUCTION}

We study a true coalition formation of three robots, i.e., 2 robots vs. 1 robot, in order to detect and capture intrusions in a curved loop. Coalition is a term that indicates a union or alliance in a society of animals. Many instances of this phenomenon can be found in nature $[4,7-13,15-16,18-19,21$, $23,27-31,33,35,39-40]$, including collective mobbing by predators [10], group attacks of conspecifics to raise dominance rank [16], and coalitions among males to gain and defend receptive females [4], [12].

Few quantitative models have been developed regarding coalition formation in natural systems in the related literature [ 1 , $2,3,5,6,8,15,17,18,20,34,37]$. Recently, in [26, 41], we studied the coalition formation of robots in order to detecting intrusions using game theory inspired by the society of animals. We considered a group of 3 robots. In our model in [26, 41], we assumed that any robot tries to form a coalition with other robots paying an investment cost when it considers that it is too weak to obtain a larger probability to capture every intrusion alone. We also considered an among-individual variation in detection strength, which is defined as the length of an individual's detection region. We considered a potential cost of forming a coalition when establishing the coalition. We are interested in identifying the conditions under which a coalition forms and those under which detecting intrusions separately always pays. We concluded in $[26,41]$ that never trying to form a coalition is always an evolutionarily stable strategy (ESS) [22], and that, when the investment cost is below a threshold, always trying to form a coalition is an ESS. However, in [26, 41], we did not draw any conclusions about whether an interior ESS exists and under what conditions a true coalition forms.

In this paper, we attempt to indentify the conditions under which an interior strategy is an ESS. Based on assumptions about the model and the results of the average payoff of the

Xiannuan Liang and Yang Xiao are with Department of Computer Science, The University of Alabama, Tuscaloosa, AL 35487-0290 USA.

Prof. Yang Xiao is the corresponding author. Email: yangxiao@ieee.org. potential mutant, which are presented in [26, 41], we analyze and determine the conditions under which an interior ESS exists. In the analysis, we find that the expression of the average payoff of the potential mutant is too complex to be studied to predict whether an interior ESS exists. Therefore, based on three different cases that depend on the ratio $(V /(L \mu)$ of the moving speed $(V)$ of the robots to the product of the possible maximum value $(L)$ of the robots' detection lengths and the frequency $(\mu)$ of the occurrence of intrusions, we make three different kinds of simplifications for the expression of the potential mutant's average payoff. As we will find in the evaluation section, the simplifications are effective and accurate. Using these effective and accurate simplifications, we find out all the conditions under which an interior ESS exists.

The rest of the paper is organized as follows: Section II presents our math model. Section III presents our robot coalition game for detecting intrusions. We finally conclude this paper in Section IV.

\section{MATH MODEL}

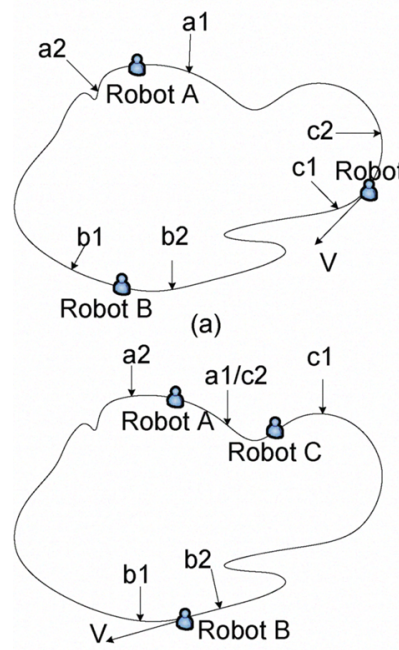

(b)
1. the length of the closed loop is $3 \mathrm{~L}$

2. the speed of each robot is $V$

3. the detect lengths of robot $A, B$, and $C$ are $\operatorname{arc}(\mathrm{a} 1, \mathrm{a} 2), \operatorname{arc}(\mathrm{b} 1, \mathrm{~b} 2)$, and $\operatorname{arc}(\mathrm{c} 1, \mathrm{c} 2)$

4. parties(robot or coalition) regularly distributed

(a): No coalition

(b): 2-way coalition vs 1 single robot

(c): 3-way coalition

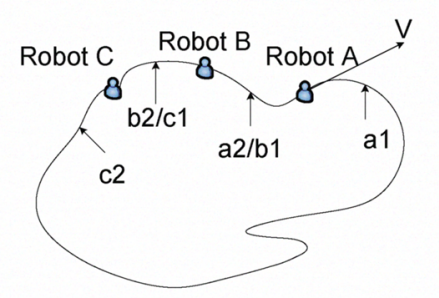

(c)
Fig. 1 Robot Coalition Scenario

We are studying the same model with the same assumptions as in $[26,41]$. In the model, we are studying the coalition formation of a group of three robots (Robots A, B, and C) that are detecting and trying to capture (or kill) intrusions in a closed loop having a length $3 L$, as shown in Fig. 1. Intrusions occur in the loop. Once an intrusion occurs, it keeps still (or lasts), for a period of time. Each robot has velocity $V$. The detection regions (lengths) of Robots A, B, and $\mathrm{C}$ are $X, Y$, and $Z$, respectively. $X, Y$, and $Z$ are random variables with the same uniform probability density function $g$ as follows: 


$$
g(x)=\left\{\begin{array}{l}
1 / L, 0<x \leq L \\
0, \text { otherwise }
\end{array}\right.
$$

The intrusion capturing/killing probability of a party (i.e., a robot or robot coalition) is defined as the product of the first intrusion detection probability of the party and the conditional capturing probability of the party. The intrusion capturing/killing probability of a group is the sum of the intrusion capturing/killing probabilities of all the parties in the group.

The assumptions for our model are as follows:

- An intrusion can be detected if it is in the detection region (length) of a robot.

- An intrusion can only be captured (killed) by the robot or robot coalition (called "a party") that detects it first.

- Intrusions occur one by one independently.

- Each intrusion occurs uniformly in the loop (i.e., each intrusion appears with probability density $1 /(3 L)$ at any point of the loop).

- Once there is an intrusion on the loop, it will last for a period of time. The length of time of each intrusion is subject to an exponential distribution having a mean $1 / \mu$ :

$$
f(x)=\left\{\begin{array}{l}
\mu e^{-\mu x}, 0<x<+\infty \\
0, \text { otherwise }
\end{array}\right.
$$

- There are time periods when there is no intrusion on the loop. Each no intrusion time period is subject to an exponential distribution having a mean $1 / \lambda$.

- A robot will try to form a coalition with other robots if its detection length is too low. The cost (called "investment cost") of the robot that seeks a coalition is $\theta \Lambda$, where the group fitness is $\Lambda$ and $0<\theta<+\infty$.

- Under the condition that a party(a coalition or a single robot) detects an intrusion first, the intrusion capturing/killing probability is: (case 1) $1 / 3$ whichever robot detects it first if there are three separate robots with no coalition, (case 2) 1 if there is a 3-way coalition, (case 3a ) $1 / 2$ if there is a 2-way coalition (or true coalition) and it is first detected by the coalition, and (case $3 b$ ) $1 / 3$ if there is a 2-way coalition (or true coalition) and it is first detected by the non-coalition robot.

- The distribution of the robots is a regular distribution (i.e., the distances between adjacent parties along the closed loop are the same if a separate robot and a coalition are both considered to be a party, as shown in Fig. 1). The total detection length of a coalition is the sum of the detection lengths of the members of the coalition. In other words, there is no overlapping between the detection regions of any pair of robots, as shown in Fig. 1.

- (Assumptions about the fitness) The probability that an intrusion can be captured or killed is 1 in the case that all three robots have detection length $L$ and form a 3-way coalition. In other cases, the probability is less than or equal to 1 . We use the maximum capturing probability to scale the group fitness and let it equal $1(\Lambda=1)$.

- (Assumptions for the allocation of the rewards) As we know that, each party has the same capturing/killing probability in any intrusion, we consider the intrusion capturing/killing probability to be the reward of the party. The allocation of the reward within the party therefore depends on the following different cases: (case 1) Each robot has its own capturing/killing probability as its reward if there is no coalition; (case 2) each robot in the party (3-way coalition) has $1 / 3$ of the capturing /killing probability of the party as its reward; (case 3) each robot in the party of the coalition has $1 / 2$ of the capturing probability of the party in the case of true coalition, and the other one has its own capturing probability as its reward.

Fig. $1 \mathrm{~b}$ shows one 2-way coalition in which the distance between any two adjacent parties is (3L- $X-Y-Z) / 2$ and the detection length of the coalition is $X+Y$.

As mentioned in one of the assumptions above, any robot will try to form a coalition with others when its detection length is too low. We call the threshold of the detection length of a robot, below which it will try to form a coalition, the strategy of the robot. As the possible range of a robot is from 0 to $L$, the possible strategy of a robot can vary from 0 to $L$.

In $[26,41]$, we worked on identifying the conditions under which a coalition of all 3 robots forms and those under which detecting intrusions separately always pays. Note that for a robot, trying to form a coalition and not trying to form a coalition are two strategies for this robot. We concluded that never trying to form a coalition (strategy 0 ) is always an evolutionarily stable strategy (ESS) [22], and that, when the investment cost is below a threshold, always trying to form a coalition (strategy L) is an ESS.

This paper deals with the following problems:

- Problem 1: Does any interior ESS (ESS between 0 and $L$ ) exist?

- Problem 2: If there is any, under what conditions does it exist?

\section{TRUe CoAlition Modeling}

To examine the existence of the interior ESS, we first need to analyze the average payoff of the potential mutant. Suppose that the strategy of the potential mutant is $w(0 \leq w \leq L)$, and that the strategies of the population (both of the other robots) are $y(0 \leq y \leq L)$. From [26, 41], we can obtain the expression of the average payoff of the potential mutant, $f(w, y)$, as follows: 


$$
\begin{aligned}
& f(w, y)=\frac{1}{L^{3}}\left(\frac{2 L^{4}+y^{3} w+w^{2} y^{2}-w^{2} y L+3 y^{2} w L}{36 L}\right. \\
& +\frac{V\left(2 L^{3}+w y^{2}-w y L\right)}{18 L \mu}-\theta w L^{2} \\
& -\frac{V}{9 L \mu} e^{-\frac{3 \mu L}{V}} \frac{V^{3}}{\mu^{3}}\left(e^{\frac{\mu}{V} w}-1\right)\left(e^{\frac{\mu}{V} y}-1\right)^{2} \\
& -\frac{V}{6 L \mu} e^{-\frac{3 \mu L}{2 V}} \frac{(2 V)^{3}}{\mu^{3}}\left(e^{\frac{\mu}{2 V} w}-1\right)\left(e^{\frac{\mu}{2 V} y}-1\right)\left(e^{\frac{\mu}{2 V} L}-e^{\frac{\mu}{2 V} y}\right) \\
& -\frac{V}{9 L \mu} e^{-\frac{3 \mu L}{2 V}} \frac{(2 V)^{3}}{\mu^{3}}\left(e^{\frac{\mu}{2 V} L}-e^{\frac{\mu}{2 V} w}\right)\left(e^{\frac{\mu}{2 V} y}-1\right)^{2} \\
& -\frac{V}{9 L \mu} e^{-\frac{\mu L}{V}} \frac{(3 V)^{3}}{\mu^{3}}\left(e^{\frac{\mu}{3 V} w}-1\right)\left(e^{\frac{\mu}{3 V} L}-e^{\frac{\mu}{3 V} y}\right)^{2} \\
& -\frac{V}{9 L \mu} e^{-\frac{\mu L}{V}} \frac{(3 V)^{3}}{\mu^{3}}\left(e^{\frac{\mu}{3 V} L}-e^{\frac{\mu}{3 V} w}\right)\left(e^{\frac{\mu}{3 V} L}+e^{\frac{\mu}{3 V} y}-2\right) \\
& \left.\left(e^{\frac{\mu}{3 V} L}-e^{\frac{\mu}{V V} y}\right)\right)
\end{aligned}
$$

The expression of $f(w, y)$ seems to be too complex for us to examine the existence of interior ESS's. We will first try to simplify the expression $f(w, y)$ to a quadratic function of $w$ using Taylor expansions and then evaluate the effect and the accuracy of the simplification.

In order to apply the Taylor expansions properly to $f(w, y)$, we need to turn back to the expression of $f(w, y)$ and use integrals. From $[26,41]$, we can easily obtain that:

$$
\begin{aligned}
& f(w, y)=\frac{1}{L^{3}}\left(\frac{2 L^{4}+w y^{3}+w^{2} y^{2}-w^{2} y L+3 y^{2} w L}{36 L}\right. \\
& \left.+\frac{V\left(2 L^{3}+w y^{2}-w y L\right)}{18 L \mu}-\theta w L^{2}\right) \\
& -\int_{1-\frac{w}{L}}^{1} \int_{1-\frac{y}{L}}^{1} \int_{1-\frac{y}{L}}^{1}\left(\frac{V}{9 L \mu}\right) e^{-\frac{\mu L\left(t_{1}+t_{2}+t_{3}\right)}{V}} d t_{1} d t_{2} d t_{3} \\
& -\int_{1-\frac{w}{L}}^{1} \int_{0}^{1-\frac{y}{L}} \int_{1-\frac{y}{L}}^{1}\left(\frac{V}{6 L \mu}\right) e^{-\frac{\mu L\left(t_{1}+t_{2}+t_{3}\right)}{2 V}} d t_{1} d t_{2} d t_{3} \\
& -\int_{1-\frac{w}{L}}^{1} \int_{0}^{1-\frac{y}{L} 1-\frac{y}{L}} \int_{0}^{L}\left(\frac{V}{9 L \mu}\right) e^{-\frac{\mu L\left(t_{1}+t_{2}+t_{3}\right)}{3 V}} d t_{1} d t_{2} d t_{3} \\
& 1-\frac{w}{L} \\
& -\int_{0}^{1} \int_{1-\frac{y}{L}}^{1} \int_{\frac{y}{L}}^{1}\left(\frac{V}{9 L \mu}\right)\left(e^{-\frac{\mu L\left(t_{1}+t_{2}+t_{3}\right)}{2 V}}-e^{-\frac{\mu L\left(t_{1}+t_{2}+t_{3}\right)}{3 V}}\right) d t_{1} d t_{2} d t_{3} \\
& 1-\frac{w}{L} 1 \\
& -\int_{0}^{1} \int_{0}^{1} \int_{0}^{1}\left(\frac{V}{9 L \mu}\right)\left(e^{-\frac{\mu L\left(t_{1}+t_{2}+t_{3}\right)}{3 V}}\right) d t_{1} d t_{2} d t_{3}
\end{aligned}
$$

Next, we inspect the integrals in expression (4) above. Then we derive three different kinds of simplification based on how large the value of $V /(L \mu)$ is. We concluded these simplifications in Lemmas 1-3. In the proofs (omitted) of Lemmas 1-3, we first examine the first integral in (4) and derive a simplification using Taylor expansion. Since the similarity between this integral and the rest of the integrals in (4) is obvious, we can apply the same simplification to the rest of them.

Lemma 1: When $V /(L \mu)$ is very small, i.e., close to 0 , we have $f(w, y) \approx a w^{2}+b w+c$, where $a=\frac{y^{2}-y L}{36 L^{4}}<0$, $b=\frac{3 y^{2} L+y^{3}}{36 L^{4}}+\frac{V\left(y^{2}-y L\right)}{18 L^{4} \mu}-\frac{\theta}{L}$, and $c=\frac{1}{18}+\frac{V}{9 L \mu}$.

Lemma 2: when $V /(L \mu)$ is very great or $(L \mu) / V$ is close to 0 , we have $f(w, y) \approx \underline{a} w^{2}+\underline{b} w+\underline{c}$, where

$$
\begin{aligned}
& \underline{a}=\frac{y^{2}-y L}{36 L^{4}}-\left(\frac{V^{2}}{18 \mu^{2} L^{4}}\right)\left(e^{-\frac{\mu(L-y)}{V}}-e^{-\frac{\mu L}{V}}\right)^{2} \\
& -\left(\frac{V^{2}}{6 \mu^{2} L^{4}}\right)\left(e^{-\frac{\mu(L-y)}{2 V}}-e^{-\frac{\mu L}{2 V}}\right)\left(1-e^{-\frac{\mu(L-y)}{2 V}}\right)-\left(\frac{V^{2}}{6 \mu^{2} L^{4}}\right)\left(1-e^{-\frac{\mu(L-y)}{3 V}}\right)^{2}, \\
& +\left(\frac{V^{2}}{9 \mu^{2} L^{4}}\right)\left(e^{-\frac{\mu(L-y)}{2 V}}-e^{-\frac{\mu L}{2 V}}\right)^{2}-\left(\frac{V^{2}}{6 \mu^{2} L^{4}}\right)\left(e^{-\frac{\mu(L-y)}{3 V}}-e^{-\frac{\mu L}{3 V}}\right)^{2} \\
& +\left(\frac{V^{2}}{6 \mu^{2} L^{4}}\right)\left(1-e^{-\frac{\mu L}{3 V}}\right)^{2} \\
& \underline{b}=\left(\frac{\left(3 y^{2} L+y^{3}\right)}{36 L^{4}}+\frac{V\left(y^{2}-y L\right)}{18 L^{4} \mu}-\frac{\theta}{L}\right) \\
& +\left[\left(\frac{V^{2}}{9 \mu^{2} L^{3}}-\frac{V^{3}}{9 \mu^{3} L^{4}}\right)\left(e^{-\frac{\mu(L-y)}{V}}-e^{-\frac{\mu L}{V}}\right)^{2}\right] \\
& +\left[\left(\frac{V^{2}}{3 \mu^{2} L^{3}}-\frac{2 V^{3}}{3 \mu^{3} L^{4}}\right)\left(e^{-\frac{\mu(L-y)}{2 V}}-e^{-\frac{\mu L}{2 V}}\right)\left(1-e^{-\frac{\mu(L-y)}{2 V}}\right)\right] \text {, and } \\
& +\left[\left(\frac{V^{2}}{3 \mu^{2} L^{3}}-\frac{V^{3}}{\mu^{3} L^{4}}\right)\left(1-e^{-\frac{\mu(L-y)}{3 V}}\right)^{2}\right] \\
& +\left[\left(\frac{4 V^{3}}{9 \mu^{3} L^{4}}-\frac{2 V^{2}}{9 \mu^{2} L^{3}}\right)\left(e^{-\frac{\mu(L-y)}{2 V}}-e^{-\frac{\mu L}{2 V}}\right)^{2}\right] \\
& +\left[\left(\frac{V^{2}}{3 \mu^{2} L^{3}}-\frac{V^{3}}{\mu^{3} L^{4}}\right)\left(e^{-\frac{\mu(L-y)}{3 V}}-e^{-\frac{\mu L}{3 V}}\right)^{2}\right] \\
& +\left[\left(\frac{V^{3}}{\mu^{3} L^{4}}-\frac{V^{2}}{3 \mu^{2} L^{3}}\right)\left(1-e^{-\frac{\mu L}{3 V}}\right)^{2}\right] \\
& \underline{c}=\left(\frac{1}{18}+\frac{V}{9 L \mu}\right)+\left[\left(\frac{V^{2}}{9 \mu^{2} L^{2}}-\frac{4 V^{3}}{9 \mu^{3} L^{3}}\right)\left(e^{-\frac{\mu(L-y)}{2 V}}-e^{-\frac{\mu L}{2 V}}\right)^{2}\right] \\
& +\left[\left(\frac{V^{3}}{\mu^{3} L^{3}}-\frac{V^{2}}{6 \mu^{2} L^{2}}\right)\left(e^{-\frac{\mu(L-y)}{3 V}}-e^{-\frac{\mu L}{3 V}}\right)^{2}\right]+\left[\left(\frac{V^{2}}{6 \mu^{2} L^{2}}-\frac{V^{3}}{\mu^{3} L^{3}}\right)\left(1-e^{-\frac{\mu L}{3 V}}\right)^{2}\right]
\end{aligned}
$$

Lemma 3: when $V /(L \mu)$ is neither very large nor very small, we have $f(w, y) \approx \bar{a} w^{3}+\bar{b} w^{2}+\bar{c} w+\bar{d}$, where

$$
\begin{aligned}
& \bar{a}=-\left(\frac{V}{54 \mu L^{4}}\right)\left(e^{-\frac{\mu(L-y)}{V}}-e^{-\frac{\mu L}{V}}\right)^{2} \\
& -\left(\frac{V}{36 \mu L^{4}}\right)\left(e^{-\frac{\mu(L-y)}{2 V}}-e^{-\frac{\mu L}{2 V}}\right)\left(1-e^{-\frac{\mu(L-y)}{2 V}}\right) \\
& -\left(\frac{V}{54 \mu L^{4}}\right)\left(1-e^{-\frac{\mu(L-y)}{3 V}}\right)^{2}+\left(\frac{V}{54 \mu L^{4}}\right)\left(e^{-\frac{\mu(L-y)}{2 V}}-e^{-\frac{\mu L}{2 V}}\right)^{2} \\
& -\left(\frac{V}{54 \mu L^{4}}\right)\left(e^{-\frac{\mu(L-y)}{3 V}}-e^{-\frac{\mu L}{3 V}}\right)^{2}+\left(\frac{V}{54 \mu L^{4}}\right)\left(1-e^{-\frac{\mu L}{3 V}}\right)^{2}
\end{aligned}
$$




$$
\begin{aligned}
& \bar{b}=\frac{y^{2}-y L}{36 L^{4}}+\left(\frac{V}{18 \mu L^{3}}-\frac{V^{2}}{18 \mu^{2} L^{4}}\right)\left(e^{-\frac{\mu(L-y)}{V}}-e^{-\frac{\mu L}{V}}\right)^{2} \\
& +\left(\frac{V}{12 \mu L^{3}}-\frac{V^{2}}{6 \mu^{2} L^{4}}\right)\left(e^{-\frac{\mu(L-y)}{2 V}}-e^{-\frac{\mu L}{2 V}}\right)\left(1-e^{-\frac{\mu(L-y)}{2 V}}\right) \\
& +\left(\frac{V}{18 \mu L^{3}}-\frac{V^{2}}{6 \mu^{2} L^{4}}\right)\left(1-e^{-\frac{\mu(L-y)}{3 V}}\right)^{2} \\
& +\left(\frac{V^{2}}{9 \mu^{2} L^{4}}-\frac{V}{18 \mu L^{3}}\right)\left(e^{-\frac{\mu(L-y)}{2 V}}-e^{-\frac{\mu L}{2 V}}\right)^{2} \\
& +\left(\frac{V}{18 \mu L^{3}}-\frac{V^{2}}{6 \mu^{2} L^{4}}\right)\left(e^{-\frac{\mu(L-y)}{3 V}}-e^{-\frac{\mu L}{3 V}}\right)^{2} \\
& +\left(\frac{V^{2}}{6 \mu^{2} L^{4}}-\frac{V}{18 \mu L^{3}}\right)\left(1-e^{-\frac{\mu L}{3 V}}\right)^{2} \\
& \bar{c}=\left(\frac{\left(3 y^{2} L+y^{3}\right)}{36 L^{4}}+\frac{V\left(y^{2}-y L\right)}{18 L^{4} \mu}-\frac{\theta}{L}\right) \\
& +\left[\left(\frac{V^{2}}{9 \mu^{2} L^{3}}-\frac{V^{3}}{9 \mu^{3} L^{4}}-\frac{V}{18 \mu L^{2}}\right)\left(e^{-\frac{\mu(L-y)}{V}}-e^{-\frac{\mu L}{V}}\right)^{2}\right] \\
& +\left[\left(\frac{V^{2}}{3 \mu^{2} L^{3}}-\frac{2 V^{3}}{3 \mu^{3} L^{4}}-\frac{V}{12 \mu L^{2}}\right)\left(e^{-\frac{\mu(L-y)}{2 V}}-e^{-\frac{\mu L}{2 V}}\right)\left(1-e^{-\frac{\mu(L-y)}{2 V}}\right)\right] \text {, and } \\
& +\left[\left(\frac{V^{2}}{3 \mu^{2} L^{3}}-\frac{V^{3}}{\mu^{3} L^{4}}-\frac{V}{18 \mu L^{2}}\right)\left(1-e^{-\frac{\mu(L-y)}{3 V}}\right)^{2}\right] \\
& +\left[\left(\frac{4 V^{3}}{9 \mu^{3} L^{4}}-\frac{2 V^{2}}{9 \mu^{2} L^{3}}+\frac{V}{18 \mu L^{2}}\right)\left(e^{-\frac{\mu(L-y)}{2 V}}-e^{-\frac{\mu L}{2 V}}\right)^{2}\right] \\
& +\left[\left(\frac{V^{2}}{3 \mu^{2} L^{3}}-\frac{V^{3}}{\mu^{3} L^{4}}-\frac{V}{18 \mu L^{2}}\right)\left(e^{-\frac{\mu(L-y)}{3 V}}-e^{-\frac{\mu L}{3 V}}\right)^{2}\right] \\
& +\left[\left(\frac{V^{3}}{\mu^{3} L^{4}}-\frac{V^{2}}{3 \mu^{2} L^{3}}+\frac{V}{18 \mu L^{2}}\right)\left(1-e^{-\frac{\mu L}{3 V}}\right)^{2}\right] \\
& \bar{d}=\left(\frac{1}{18}+\frac{V}{9 L \mu}\right) \\
& +\left[\left(\frac{V^{2}}{9 \mu^{2} L^{2}}-\frac{4 V^{3}}{9 \mu^{3} L^{3}}-\frac{V}{54 \mu L}\right)\left(e^{-\frac{\mu(L-y)}{2 V}}-e^{-\frac{\mu L}{2 V}}\right)^{2}\right] . \\
& +\left[\left(\frac{V^{3}}{\mu^{3} L^{3}}-\frac{V^{2}}{6 \mu^{2} L^{2}}+\frac{V}{54 \mu L}\right)\left(e^{-\frac{\mu(L-y)}{3 V}}-e^{-\frac{\mu L}{3 V}}\right)^{2}\right] \\
& +\left[\left(\frac{V^{2}}{6 \mu^{2} L^{2}}-\frac{V^{3}}{\mu^{3} L^{3}}-\frac{V}{54 \mu L}\right)\left(1-e^{-\frac{\mu L}{3 V}}\right)^{2}\right]
\end{aligned}
$$

After obtaining the approximate expressions of $f(w, y)$ by quadratic or cubic simplification in the three different cases, we can conclude the following three theorems for the three different cases. Let $f(w, y, z)$ denote the pay-off of the potential mutant with a strategy $w$ versus an individual with strategy $y$ and another with strategy $z$. Note that $f(w, y)$ is the pay-off of the potential mutant with a strategy $w$ versus a population of the other two individuals both with strategy $y$. We have $f(w, y)=f(w, y, y)$. Both notations of $f(w, y)$ and $f(w, y, z)$ appearing in the theorems themselves and the proofs of the theorems are regarded to be the approximations of the payoffs after simplification.

Theorem 1: When $V /(L \mu)$ is very small (the case of Lemma 1), according to the notations above, we have the following conclusion about whether $y, 0<y<L$, is an interior ESS: 1) when $-b /(2 a)=y, y$ is an ESS; 2) when $-b /(2 a) \neq y, y$ is not an ESS.

Theorem 2: When $V /(L \mu)$ is very large (the case of Lemma2 ), according to the notations above, we have the following conclusion about whether $y, 0<y<L$, is an interior ESS: 1) when $\underline{a}>0, y$ is not an ESS; 2) when $\underline{a}<0$ and $-\underline{b} /(2 \underline{a})=y, y$ is an ESS; 3) when $\underline{a}<0$ and $-\underline{b} /(2 \underline{a}) \neq y, y$ is not an ESS; 4) when $\underline{a}=0$ and $\underline{b} \neq 0, y$ is not an ESS; 5) when $\underline{a}=0$ and $\underline{b}=0, y$ is not an ESS.

Let us define the following:

$$
\begin{aligned}
& a_{0}=-\left(\frac{V}{54 \mu L^{4}}\right)\left(1-e^{-\frac{\mu L}{V}}\right)\left(e^{-\frac{\mu(L-y)}{V}}-e^{-\frac{\mu L}{V}}\right) \\
& -\left(\frac{V}{72 \mu L^{4}}\right)\left(1-e^{-\frac{\mu L}{2 V}}\right)\left(1-e^{-\frac{\mu(L-y)}{2 V}}\right) \\
& +\left(\frac{V}{54 \mu L^{4}}\right)\left(1-e^{-\frac{\mu L}{2 V}}\right)\left(e^{-\frac{\mu(L-y)}{2 V}}-e^{-\frac{\mu L}{2 V}}\right), \\
& -\left(\frac{V}{54 \mu L^{4}}\right)\left(1-e^{-\frac{\mu L}{2 V}}\right)\left(e^{-\frac{\mu(L-y)}{2 V}}-e^{-\frac{\mu L}{2 V}}\right) \\
& +\left(\frac{V}{54 \mu L^{4}}\right)\left(1-e^{-\frac{\mu L}{3 V}}\right)^{2} \\
& b_{0}=\frac{(2 y-L)}{72 L^{3}} \\
& +\left(\frac{V}{18 \mu L^{3}}-\frac{V^{2}}{18 \mu^{2} L^{4}}\right)\left(1-e^{-\frac{\mu L}{V}}\right)\left(e^{-\frac{\mu(L-y)}{V}}-e^{-\frac{\mu L}{V}}\right) \\
& +\left(\frac{V}{24 \mu L^{3}}-\frac{V^{2}}{12 \mu^{2} L^{4}}\right)\left(1-e^{-\frac{\mu L}{2 V}}\right)\left(1-e^{-\frac{\mu(L-y)}{2 V}}\right) \\
& +\left(\frac{V^{2}}{9 \mu^{2} L^{4}}-\frac{V}{18 \mu L^{3}}\right)\left(1-e^{-\frac{\mu L}{2 V}}\right)\left(e^{-\frac{\mu(L-y)}{2 V}}-e^{-\frac{\mu L}{2 V}}\right), \\
& +\left(\frac{V}{18 \mu L^{3}}-\frac{V^{2}}{6 \mu^{2} L^{4}}\right)\left(1-e^{-\frac{\mu L}{2 V}}\right)\left(e^{-\frac{\mu(L-y)}{2 V}}-e^{-\frac{\mu L}{2 V}}\right) \\
& +\left(\frac{V^{2}}{6 \mu^{2} L^{4}}-\frac{V}{18 \mu L^{3}}\right)\left(1-e^{-\frac{\mu L}{3 V}}\right)^{2} \\
& \left.c_{0}=\frac{\left(L y+4 y^{2}+3 L^{2}\right)}{72 L^{3}}+\frac{V(y-L)}{36 L^{3} \mu}-\frac{\theta}{L}\right) \\
& +\left[\left(\frac{V^{2}}{9 \mu^{2} L^{3}}-\frac{V^{3}}{9 \mu^{3} L^{4}}-\frac{V}{18 \mu L^{2}}\right)\left(1-e^{-\frac{\mu L}{V}}\right)\left(e^{-\frac{\mu(L-y)}{V}}-e^{-\frac{\mu L}{V}}\right)\right] \\
& +\left[\left(\frac{V^{2}}{6 \mu^{2} L^{3}}-\frac{V^{3}}{3 \mu^{3} L^{4}}-\frac{V}{24 \mu L^{2}}\right)\left(1-e^{-\frac{\mu L}{2 V}}\right)\left(1-e^{-\frac{\mu(L-y)}{2 V}}\right)\right] \\
& +\left[\left(\frac{4 V^{3}}{9 \mu^{3} L^{4}}-\frac{2 V^{2}}{9 \mu^{2} L^{3}}+\frac{V}{18 \mu L^{2}}\right)\left(1-e^{-\frac{\mu L}{2 V}}\right)\left(e^{-\frac{\mu(L-y)}{2 V}}-e^{-\frac{\mu L}{2 V}}\right)\right] \\
& +\left[\left(\frac{V^{2}}{3 \mu^{2} L^{3}}-\frac{V^{3}}{\mu^{3} L^{4}}-\frac{V}{18 \mu L^{2}}\right)\left(1-e^{-\frac{\mu L}{2 V}}\right)\left(e^{-\frac{\mu(L-y)}{2 V}}-e^{-\frac{\mu L}{2 V}}\right)\right] \\
& +\left[\left(\frac{V^{3}}{\mu^{3} L^{4}}-\frac{V^{2}}{3 \mu^{2} L^{3}}+\frac{V}{18 \mu L^{2}}\right)\left(1-e^{-\frac{\mu L}{3 V}}\right)^{2}\right]
\end{aligned}
$$




$$
\begin{aligned}
& d_{0}=\frac{1}{18}+\frac{V}{9 L \mu} \\
& +\left[\left(\frac{V^{2}}{9 \mu^{2} L^{2}}-\frac{4 V^{3}}{9 \mu^{3} L^{3}}-\frac{V}{54 \mu L}\right)\left(1-e^{-\frac{\mu L}{2 V}}\right)\left(e^{-\frac{\mu(L-y)}{2 V}}-e^{-\frac{\mu L}{2 V}}\right)\right] \\
& +\left[\left(\frac{V^{3}}{\mu^{3} L^{3}}-\frac{V^{2}}{6 \mu^{2} L^{2}}+\frac{V}{54 \mu L}\right)\left(1-e^{-\frac{\mu L}{2 V}}\right)\left(e^{-\frac{\mu(L-y)}{2 V}}-e^{-\frac{\mu L}{2 V}}\right)\right] \\
& +\left[\left(\frac{V^{2}}{6 \mu^{2} L^{2}}-\frac{V^{3}}{\mu^{3} L^{3}}-\frac{V}{54 \mu L}\right)\left(1-e^{-\frac{\mu L}{3 V}}\right)^{2}\right]
\end{aligned}
$$

Theorem 3: When $V /(L \mu)$ is neither large nor small (the same case of Lemma 3), and when $x_{1}=\frac{-2 \bar{b}}{6 \bar{a}}-\frac{\sqrt{4 \bar{b}^{2}+12 \overline{a c}}}{6|\bar{a}|}$, $x_{2}=\frac{-2 \bar{b}}{6 \bar{a}}+\frac{\sqrt{4 \bar{b}^{2}+12 \overline{a c}}}{6|\bar{a}|} \quad, \quad x_{3}=\frac{-2 b_{0}}{6 a_{0}}-\frac{\sqrt{4 b_{0}^{2}+12 a_{0} c_{0}}}{6\left|b_{0}\right|}$, $x_{4}=\frac{-2 b_{0}}{6 a_{0}}+\frac{\sqrt{4 b_{0}^{2}+12 a_{0} c_{0}}}{6\left|b_{0}\right|}, x_{5}=\frac{-2 b_{1}}{6 a_{1}}-\frac{\sqrt{4 b_{1}^{2}+12 a_{1} c_{1}}}{6\left|a_{1}\right|}$, and $x_{6}=\frac{-2 b_{1}}{6 a_{1}}+\frac{\sqrt{4 b_{1}^{2}+12 a_{1} c_{1}}}{6\left|a_{1}\right|}$, we have the following conclusions about whether $y, 0<y<L$, is an interior ESS.

- When $\bar{a}=0$ :

○ If $\bar{b}<0$, and $2 \bar{b} y+\bar{c}=0, y$ is an ESS.

- If $\bar{b}<0$, and $2 \bar{b} y+\bar{c} \neq 0, y$ is not an ESS.

- If $\bar{b}>0, y$ is not an ESS.

○ If $\bar{b}=0, y$ is not an ESS.

- When $\bar{a}>0$, and $4 \bar{b}^{2}+12 \overline{a c} \leq 0, y$ is not an ESS.

- When $\bar{a}>0$, and $4 \bar{b}^{2}+12 \overline{a c}>0$ :

○ If $x_{1} \neq y, y$ is not an ESS.

○ If $x_{1}=y$, and $f(y, y)>f(L, y), y$ is an ESS.

○ If $x_{1}=y$, and $f(y, y)<f(L, y), y$ is not an ESS.

○ If $x_{1}=y$, and $f(y, y)=f(L, y)$, to determine whether $y$ is an ESS, we need to apply this theorem again to the function $f(h, L, y)$ of $y: f(h, L, y)=a_{0} h^{3}+b_{0} h^{2}+c_{0} h+d_{0}$ :

- If $a_{0}=0, b_{0}<0$, and $2 b_{0} y+c_{0}=0, y$ is an ESS;

- If $a_{0}>0,4 b_{0}{ }^{2}+12 a_{0} c_{0}>0, \bar{x}_{3}=y$, and $f(y, L, y)>f(L, L, y), y$ is an ESS;

- If $a_{0}<0,4 b_{0}{ }^{2}+12 a_{0} c_{0}>0, \bar{x}_{4}=y$, and $f(y, L, y)>f(0, L, y), y$ is an ESS;

- Otherwise, $y$ is not an ESS.

- When $\bar{a}<0$, and $4 \bar{b}^{2}+9 \overline{a c} \leq 0, y$ is not an ESS.

- When $\bar{a}<0$, and $4 \bar{b}^{2}+9 \overline{a c}>0$ :

○. If $x_{2} \neq y, y$ is not an ESS.
- If $x_{2}=y$, and $f(y, y)>f(0, y), y$ is an ESS.

- If $x_{2}=y$, and $f(y, y)<f(0, y), y$ is not an ESS.

○ If $x_{2}=y$, and $f(y, y)=f(0, y)$, to determine whether $y$ is an ESS, we need to apply this theorem again to the function $f(h, 0, y)$ of $h: f(h, 0, y)=a_{1} h^{3}+b_{1} h^{2}+c_{1} h+d_{1}$ :

- If $a_{1}=0, b_{1}<0$, and $2 b_{1} y+c_{1}=0, y$ is an ESS;

- If $a_{1}>0,4 b_{1}^{2}+12 a_{1} c_{1}>0, \bar{x}_{5}=y$, and $f(y, 0, y)>f(L, 0, y), y$ is an ESS;

- If $a_{1}<0,4 b_{1}^{2}+12 a_{1} c_{1}>0, \bar{x}_{6}=y$, and $f(y, 0, y)>f(0,0, y), y$ is an ESS;

- Otherwise, $y$ is not an ESS.

Next, we consider the probability of true coalition formation. In a population at an ESS, $y$, there are 3 possible outcomes for every triad that draws 3 detection lengths from the distribution. The probability that a 3-way coalition forms is

$$
\begin{aligned}
& P_{1}(y)=\operatorname{Pr} o b(X<y, Y<y, Z<y)=(1-G(y))^{3}, \text { where } \\
& G(y)=\int_{0}^{y} \frac{1}{L} d x=\frac{y}{L} .
\end{aligned}
$$

The probability that no coalition forms is

$$
\begin{aligned}
& P_{2}(y)=\operatorname{Pr} o b(X \geq y, Y \geq y, Z \geq y) \\
& +3 \operatorname{Pr} o b(X \geq y, Y \geq y, Z<y) \\
& =(G(y))^{3}+3(G(y))(1-G(y))^{2}
\end{aligned} .
$$
is

The probability that a true coalition (2-way coalition) forms

$$
P_{3}(y)=1-P_{1}(y)-P_{2}(y)=3(G(y))^{2}(1-G(y)) .
$$

$P_{3}(y)$ is zero when $y=0$ or $y=1$. The probability of a true alliance is non-zero only when $0<y<L$ (i.e., when $y$ is an interior ESS).

\section{CONCLUSION}

In our model, we have drawn the conclusion that the formation of a true coalition is possible only when an interior ESS exists. Depending on the three value range of $V /(L \mu)$, we use three kinds of simplification of the payoff of the potential mutant, $f(w, y)$. In the evaluation section, we have examined the effect of the simplifications and have found that the simplifications are accurate. Using these simplifications, we have derived that, in certain conditions, an interior ESS exists.

\section{ACKNOWLEDGEMENT}

The work was supported in part by the US National Science Foundation (NSF) under the grant numbers CCF-0829827, CNS-0716211, and CNS-0737325.

\section{REFERENCES}

[1] S. A. Pandit and C. P. van Schaik, "A model for leveling coalitions among primate males: toward a theory of egalitarianism," Behav Ecol Sociobiol. Vol. 55, pp. 161-168, 2003. 
[2] R. Noe, "A model of coalition formation among male baboons with fighting ability as the crucial parameter," Anim Behav. 47:211-213. Noe" R, Sluijter AA. 1995. Which adult male savanna baboons form coalitions Int J Primatol. Vol. 16, pp. 77-105. 1994.

[3] L. A. Dugatkin, "A model of coalition formation in animals," Proc R Soc Lond B Biol Sci. Vol. 265, pp. 2121-2125. 1998.

[4] C. Packer, D. A. Gilbert, A. E. Pusey, and S. J. O'Brien, "A molecular genetic analysis of kinship and co-operation in African lions," Nature, Vol. 351, pp. 562-565, 1991.

[5] R. Noe, "A veto game played by baboons: a challenge to the use of the prisoner's dilemma as a paradigm for reciprocity and cooperation," Anim Behav. Vol. 39, pp. 78-90, 1990.

[6] H. Whitehead and R. Connor, "Alliances II. How large should alliances be?" Anim Behav. Vol. 69, pp. 117-126, 2005.

[7] R. Connor and H. Whitehead, "Alliances II. Rates of encounter during resource utilization: a general model of intrasexual alliance formation in fission-fusion societies," Anim Behav. Vol. 69, pp. 127-132, 2005.

[8] R. Noe, "Alliance formation among male baboons: shopping for profitable partners," In: Harcourt AH, de Waal FBM, editors. Coalitions and alliances in humans and other animals. Oxford: Oxford University Press. pp. 285-321. 1992.

[9] M. Mesterton-Gibbons, "An introduction to game-theoretic modeling," 2001.

[10] T. Caro, "Antipredator defenses in birds and mammals," Chicago:University of Chicago Press, 2005.

[11] L. A. Dugatkin. "Breaking up fights between others: a model of intervention behaviour," Proc R Soc Lond B Biol Sci. Vol. 265, pp. 433-436, 1998.

[12] T. M. Caro, "Cheetahs of the Serengeti Plains: group living in an asocial species," Wildlife Behaviour and Ecology. Chicago: University of Chicago Press. 1994

[13] F.B. M. de Waal and A. H. Harcourt AH, "Coalitions and alliances: a history of ethological research," In: Harcourt AH, de Waal FBM, editors. Coalitions and alliances in humans and other animals. Oxford: Oxford University Press. pp. 1-19, 1992.

[14] F. B. Bercovitch, "Coalitions, cooperation, and reproductive tactics among adult male baboons," Anim Behav. Vol. 36, pp. 1198-1209, 1988.

[15] M. Mesterton-Gibbons and T. N. Sherratt, "Coalition formation: a game-theoretic analysis," Behavioral Ecology. Vol. 18, No. 2, Mar. 2007, pp. 277-286.

[16] C. J. Zabel, S. E. Glickman, L. G. Frank, K. B. Woodmansee, and G. Keppel, "Coalition formation in a colony of prepubertal spotted hyaenas," In: Harcourt AH, de Waal FBM, editors. Coalitions and alliances in humans and other animals. Oxford: Oxford University Press. p. 113-135. 1992.

[17] R. A. Johnstone and L. A. Dugatkin LA, "Coalition formation in animals and the nature of winner and loser effects," Proc R Soc Lond B Biol Sci. Vol. 267, pp. 17-21, 2000

[18] R. C. Connor, R. A. Smolker, A. F. Richards, "Dolphin alliances and coalitions," In: Harcourt AH, de Waal FBM, editors. Coalitions and alliances in humans and other animals. Oxford: Oxford University Press. p. 415-443, 1992.

[19] J. M. Smith, "Evolution and the theory of games," Cambridge (UK): Cambridge University Press, 1982.
[20] M. Enquist and O. Leimar, "Evolution of fighting behaviour: decision rules and assessment of relative strength," J Theor Biol. Vol. 102, pp. 387-410, 1983.

[21] I, Eshel, "Evolutionary and continuous stability," J Theor Biol. Vol. 103, pp. 99-111, 1983.

[22] http://en.wikipedia.org/wiki/Evolutionary stable strategy

[23] L. J. Morrell, P.R. Y. Backwell, and N. B. Metcalfe, "Fighting in fiddler crabs Uca mjoebergi: what determines duration?" Anim Behav. Vol. 70, pp. 653-662, 2005.

[24] L. A Dugatkin, "Game theory and cooperation," In: Dugatkin LA, Reeve HK, editors. Game theory and animal behavior. New York: Oxford University Press. pp. 38-63, 1998.

[25] C. Boehm, "Hierarchy in the forest: the evolution of egalitarian behavior," Cambridge (MA): Harvard University Press. 1999.

[26] X. Liang and Y. Xiao, "Modeling Collaborative Robots for Detecting Intrusions," Proceedings of The 2009 International Symposium on Collaborative Technologies and Systems (CTS 2009), pp.306-313.

[27] J. N. McDonald, "North American bison," Berkeley (CA): University of California Press, 1981

[28] F. B. Christiansen, "On conditions for evolutionary stability for a continuously varying character," Am Nat. Vol. 138, pp. 37-50, 1991.

[29] A. L. Engh, E. R. Siebert, D. A. Greenberg, and K. E. Holekamp, "Patterns of alliance formation and postconflict aggression indicate spotted hyaenas recognise third-party relationships," Anim Behav. Vol. 69, pp. 209-217, 2005.

[30] J. B. Silk, S. C. Alberts, and J. Altmann, "Patterns of coalition formation by adult female baboons," in Amboseli, Kenya. Anim Behav. Vol. 67, pp. 573-582, 2004.

[31] O. Kempthorne and L. Folks, "Probability, statistics, and data analysis," Ames (IA): The Iowa State University Press, 1971.

[32] C. Packer, "Reciprocal altruism in Papio anubis," Nature. Vol. 265, pp. 441-443, 1977.

[33] R. C. Connor, R. M. Heithaus, and L. M. Barre, "Superalliance of bottlenose dolphins," Nature. Vol. 371, pp. 571-572, 1999.

[34] C. K. Hemelrijk, "Support for being groomed in long-tailed macaques, Macaca fascicularis," Anim Behav. Vol. 48, pp.479-481, 1994.

[35] J. Moya-Larano and D. H. Wise, "Survival regression analysis: a powerful tool for evaluating fighting and assessment," Anim Behav. Vol. 60, pp. 307-313, 2000.

[36] J. M. Smith and G. R. Price, "The logic of animal conflict," Nature 246, pp. 15-18, 1973.

[37] L. Barrett and S. P. Henzi, "The utility of grooming in baboon troops," In: Noe" R, van Hooff JARAM, Hammerstein P, editors. Economics in nature: social dilemmas, mate choice and biological markets, 2001.

[38] Y. Hsu and L. L. Wolf, "The winner and loser effect: integrating multiple experiences," Anim Behav. Vol. 57, pp. 903-910, 1999.

[39] M. Bulmer, "Theoretical evolutionary ecology. Sunderland (MA):Sinauer," 1994.

[40] C. Rutte, M. Taborsky, M. W. G. Brinkhof, "What sets the odds of winning and losing?" Trends Ecol Evol. Vol. 21, pp. 16-21, 2006.

[41] X. Liang and Y. Xiao, "Studying Bio-inspired Coalition Formation of Robots for Detecting Intrusions Using Game Theory," IEEE Transactions on Systems, Man, and Cybernetics, Part B, accepted and to appear. 